\title{
AN EXPEDITIOUS AND CONSISTENT PROCEDURE FOR TETRAHYDROFURAN (THF) DRYING AND DEOXYGENATION BY THE STILL APPARATUS
}

\author{
Alessandro B. C. Simas*, Vera L. P. Pereira, Cleber B. Barreto Jr., Daniel L. de Sales e Leandro L. de Carvalho \\ Núcleo de Pesquisas de Produtos Naturais, Centro de Ciências da Saúde, bl. H, Universidade Federal do Rio de Janeiro, Ilha do \\ Fundão, 21941-902 Rio de Janeiro - RJ, Brasil
}

Recebido em 2/4/09; aceito em 26/5/09; publicado na web em 20/10/09

\begin{abstract}
Despite the availability of alternative methods for drying tetrahydrofuran (THF), the use of the still apparatus, wherein a THF solution containing Na-bezophenone ketyl is heated to reflux, remains widespread. We herein propose a set of procedures to solve the problems usually faced in applying this drying technique. Moreover, a discussion is made on the chemical knowledge underlying such procedures. Safety and economy issues concerned with the operation of the THF still apparatus are also discussed.
\end{abstract}

Keywords: THF; still apparatus; purification.

The demand for dry and $\mathrm{O}_{2}$-free organic solvents is an integral part of the experimental work in Chemistry. In the case of more volatile solvents, this is classically achieved by use of the still apparatus. This closed vertical distillation system (Figure 1) consists of a round-bottom flask containing a scavenging agent, a collector in the middle, a condenser, and a pressure relief system at the top. The solvent mixture is brought to reflux by using a heating mantle. In the case of ethereal solvents (THF, $\mathrm{Et}_{2} \mathrm{O}$ ), the drying/deoxygenation agent is the $\mathrm{Na}^{\circ}$-benzophenone combination. ${ }^{1}$

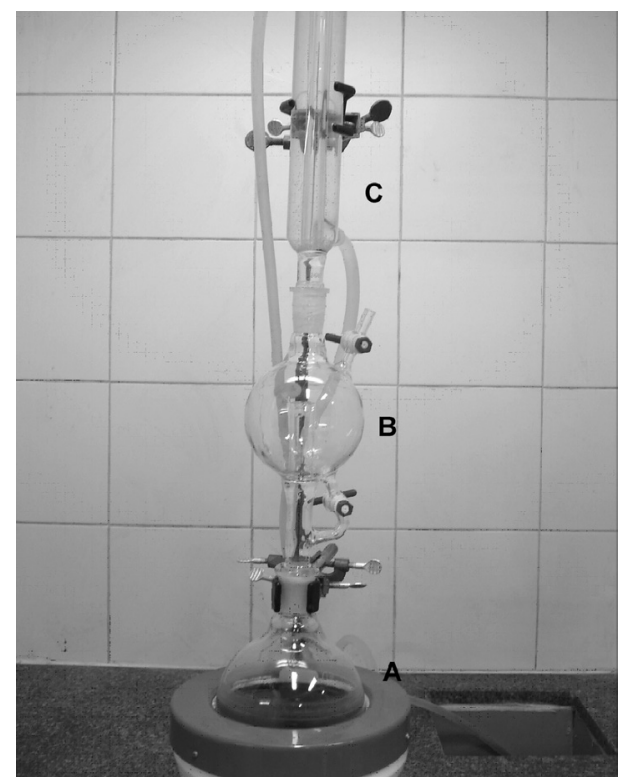

Figure 1. The still apparatus Heating mantle (A); collector (B); condenser (C)

When the solvent is essentially dry and free of $\mathrm{O}_{2}$, the Nabenzophenone ketyl species, $\mathbf{2}$ (Scheme 1), a radical anion, is formed and for that the solvent mixture turns blue (high concentration of that species). This reactive species is an effective $\mathrm{O}_{2}$-scavenging agent. A study by Mallinkrodt (now Mallinkrodt-Baker) ${ }^{2}$ established that still apparatus running on this condition affords deoxygenated THF with $\mathrm{H}_{2} \mathrm{O}$ content in the ppm range ( $\left.10 \mathrm{ppm}\right)$.

*e-mail: abcsimas@nppn.ufrj.br

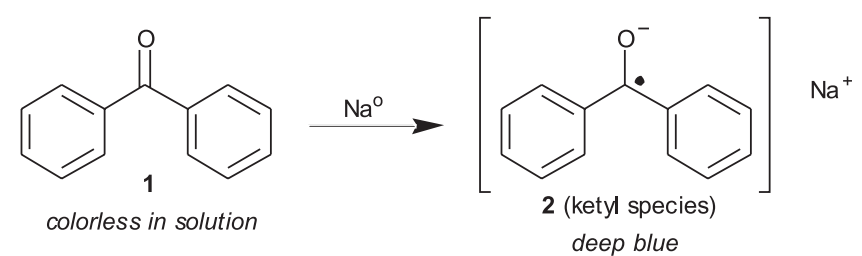

Scheme 1. The formation of Na-benzophenone ketyl species

More recently, an alternative procedure for drying solvents has been proposed by Grubbs and collaborators. ${ }^{3,4} \mathrm{By}$ this, high-quality solvents are pressurized through activated alumina (A-2) column to remove $\mathrm{H}_{2} \mathrm{O}$, peroxides and oxidation inhibitors (e.g., BHT). For hydrocarbons, a second in-line column containing a copper catalyst (Q-5) is used to scavenge $\mathrm{O}_{2}$. In the case of $\mathrm{CH}_{2} \mathrm{Cl}_{2}$, THF, $\mathrm{Et}_{2} \mathrm{O}$, etc., as these materials are not compatible with this catalyst, a second column of alumina (Optional) can replace it. In order to provide $\mathrm{O}_{2}$ free solvents, a thorough deoxygenation should thus precede their loading into this filtration system. To the best of our knowledge, this distillation-free system has been commercialized by two companies, at least, in Europe and the U.S.A..

Studies have argued that, although this apparatus is more expensive to put in place, the initial high cost is reversed over time. ${ }^{5}$ It is suggested that the classical still apparatus has a higher cost of operation than this newer one. Overall, safety issues seemingly favor the column-filtration system. ${ }^{6,7}$ In fact, for laboratories with large demand of purified solvents, the pressurized-column system should be considered, at least for the bulk of their solvent consuming.

However, it should be recognized that part of the cost of running the stills have to do with traditional misguided practices such as permanent (24/7) reflux of flammable solvent mixtures containing pyrophoric agents $\left(\mathrm{Na}^{\circ}\right.$ in the case of THF), as well as continuous supply of $\mathrm{H}_{2} \mathrm{O}$ for refrigeration. ${ }^{5}$ Most of the reported accidents involving still apparatuses may have to do with problems in the attendance of the drying process. These problems presumably raise the intrinsic hazards of the procedure. We have concluded that the 24/7 reflux is not necessary whatsoever. Thus, a daily 20-min stirring of the THF sample, after a careful purge with Ar (vide infra for details), suffices to maintain this solvent in good conditions for use. The water circulation required to condense the solvent under reflux may be effected by a closed loop system, run by means of a small pump (e.g., small aquarium pump). In fact, modern chemistry buildings, whose design 
takes environmental concerns into account, already incorporate a closed loop water circulation line. Such practices altogether make the operation of THF stills safer and more economical. Furthermore, they also demand less attendance due to the enhanced safety.

Our experience and of many others has shown that, even the continuous supply of dry $\mathrm{N}_{2}$ (through a line), may be substituted by use of a balloon (with Ar, preferentially), provided that proper care is taken (vide general recommendations).

Being a procedure that provides very dry deoxygenated THF, through a single process, affording instantaneous indication of its purity, remains the greatest advantage of the use of benzophenoneNa ketyl. Naturally, the still apparatus for THF drying poses hazard as hot vapors of a highly flammable solvent are formed during its operation, which also relies on use of pyrophoric agent. A responsible program for awareness of good practices with constant overseeing (and enforcement) by experienced chemists should necessarily be put in place.

Difficulties in making the drying process with the Na-benzophenone ketyl work promptly are common. After a first drying attempt fails, re-distillation of THF is often tried. Sometimes, the THF mixture does not turn blue after heating for days over large amounts of $\mathrm{Na}^{\circ}$ and with several additions of benzophenone. Drying THF by means of the Na-benzophenone ketyl procedures appears to be more troublesome than other ethereal solvents $\left(\mathrm{Et}_{2} \mathrm{O}\right.$, dioxane) with regard to its readiness.

Our observations on the use of THF stills led us to figure out that if few factors are taken into account and properly combined, this drying process becomes much more consistent and expeditious. A discussion on this optimized protocol follows.

For the purification process to work, it is not necessary to employ high-quality THF, ${ }^{2,3}$ with very low content of water or other impurities (e.g. HPLC grade). In any case, we submit THF, high-quality or not (ACS grade or PA grade in Brazil), to a pretreatment stage (See procedure). Reflux over $\mathrm{NaOH}$ or $\mathrm{KOH}$ for $2 \mathrm{~h}$ may substitute the phase consisting of storage over the same reagent. The employment of such pretreatment step eventually speeds up the whole drying process because it prevents the need of distillation after a failed attempt of direct drying with $\mathrm{Na}^{\circ}$. Excess of impurities formed by action of $\mathrm{Na}^{\circ}$ likely prevents the formation of benzophenone's ketyl species.

It is noteworthy that peroxides are known to rapidly decompose in the presence of bases. ${ }^{8-10}$ This process, an elimination reaction, depends on the presence of a hydrogen atom at the $\alpha$-carbon atom (Scheme 2), which is certainly the case of the impurities originated from THF. We presume that, under the pretreatment conditions, this degradation process mediated by hydroxide takes place in a more controlled fashion as peroxides undergo this reaction while in solution. That may prevent the risk of explosion due to peroxide concentration during the distillation. ${ }^{8}$

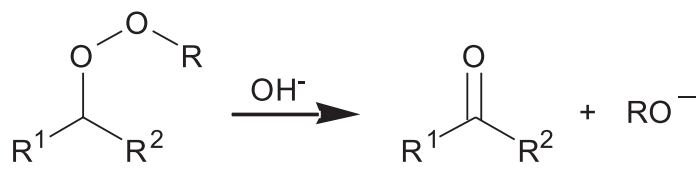

Scheme 2. Base-catalyzed degradation of peroxides

We have not experienced a single incident at this stage, even when 4-year old solvent (Closed bottles) was treated as described here. Care nonetheless has been taken not to distill THF to dryness. Old partially-used THF bottles should not be employed (Discharge is advised) provided that a specific pretreatment for elimination of peroxides is carried out. In any case, it is recommended to test for peroxides whenever older bottles of this solvent are used. ${ }^{8}$
After these preliminary steps, the actual drying of THF starts by stirring a mixture of $\mathrm{Na}^{\circ}$ and $\mathrm{THF}$ under inert atmosphere. During this time, the surface of $\mathrm{Na}^{\circ}$ is periodically cleaned to remove deposited hydroxide and oxide, what renders unnecessary the addition of excessive amounts of $\mathrm{Na}^{\circ}$. This is commonly deemed to be unavoidable to complete the drying process. The indicator, benzophenone, is only added after such condition is met. We have found that the Na-benzophenone ketyl indicator system works better when a combination of use of $\mathrm{Na}^{\circ}$ with active surface, judicious removal of air (preferentially by Ar) and, stirring is applied. The use of stirring only facilitates blue color formation if the THF-Na mixture containing the indicator has been freed of air. This explains why, by the proposed procedure, stirring is only started after this condition is secured. Actually, this is explained by the fact that the ketyl species is thought to be a strong $\mathrm{O}_{2}$ scavenger. The Na-benzophenone ketyl indicator system is set off at room temperature (Figure $1 \mathrm{~S}$, supplementary information). In fact, in our daily work, we only heat the THF still if the solvent turns blue at room temperature. Resorting to reflux, ${ }^{1}$ instead of the procedure proposed here, usually leads to a much delayed response from the indicator. This procedure also substantially cuts down the amount of $\mathrm{Na}^{\circ}$ required for drying. Whenever the blue color fades away, one should first clean $\mathrm{Na}^{\circ}$ surface, before adding a controlled amount of indicator. Use of additional $\mathrm{Na}^{\circ}$ may be necessary after continued use.

As this protocol works so well, we suppose that it is possible to employ smaller amounts of $\mathrm{Na}^{\circ}$. The recommendations just given should be successively followed up before attempting to set off the indicator system.

The study by the Mallinkrodt company mentioned before ${ }^{2}$ showed that THF stills, set up as proposed originally, provide THF containing up to $150 \mathrm{ppm}$ of benzene. The Na-benzophenone ketyl mixture was also analyzed and it was shown to contain different compounds, formed after disproportionation of this reactive species (Figure 2). Their formation clearly stems from the formation of phenyl radicals, which also lead to formation of benzene. For the most part, the presence of benzene in very low concentrations is not a problem. Most of the applications of THF as solvent in the literature have employed still apparatuses in its purification.

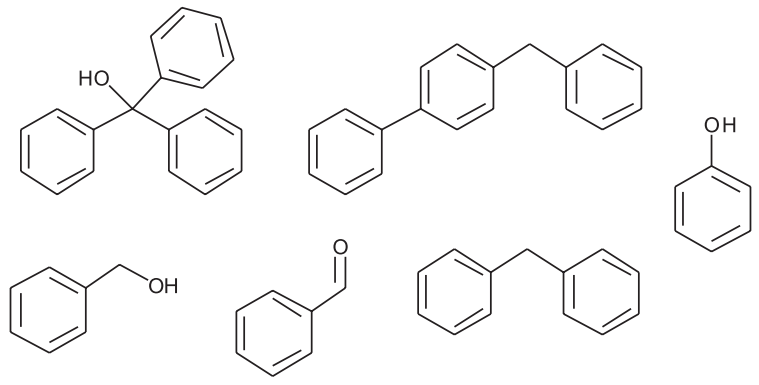

Figure 2. Substances formed after disproportionation of the Na-benzophenone ketyl species

For practical reasons, the still apparatus with the THF flask is only assembled after the solvent is found dry by the benzophenone indicator. An inert internal atmosphere is also secured before heating to reflux is started. Although it is not usually necessary to stir the THF flask before heating, we preventively do so. At last, recommendations are made to keep THF ready for use (vide experimental). As mentioned before, these render unnecessary 24/7 reflux of the THF-Na mixture.

We have found that this procedure also works with $\mathrm{Et}_{2} \mathrm{O}$. Thus, it is reasonable to anticipate that it may be successfully applied to other ethereal solvents such as, dioxane, DME, etc.. 
In conclusion, a straightforward and very consistent procedure for THF drying employing the still apparatus has been reported. The formation of benzophenone ketyl species occur quite fast at room temperature. Our experience indicates that, for the Na-benzophenone ketyl indicator system to work properly, a combination of factors are important. In fact, the procedure proposed here makes the use of large amounts of $\mathrm{Na}^{\circ}$ and benzophenone unnecessary. It is also time-saving as it avoids unfruitful attempts. As discussed above, the classical still apparatus remains useful because it provides THF free of $\mathrm{O}_{2}$ and with very low level of $\mathrm{H}_{2} \mathrm{O}$, by a very practical procedure. And this is achieved in a single operation with instantaneous assessment of quality (blue color).

\section{MATERIALS}

THF was purchased from Tedia, Merck, Vetec.

\section{General recommendations}

Operation of THF stills (including the recommended purges with Ar) and the drying protocol should be carried out in venting hoods. When THF is under reflux, this apparatus should be under supervision by a trained person. Special attention should be paid to the pressure relief (especially, as the temperature is rising) and $\mathrm{H}_{2} \mathrm{O}$ circulation. In case balloons replace the $\mathrm{N}_{2}$ line, it is recommended the use of two balloons in order to ensure that a pressure relief is actually in place. $\mathrm{Ar}$ in the balloons is periodically replaced by new Ar to avoid potential diffusion of air into them. Awareness of the risks involved and steps to be taken in case of accidents should be part of the training program. $\mathrm{Na}^{\circ}$ should be carefully quenched before disposal and clean-up of the THF flask. We usually recycle the bulk of the remaining $\mathrm{Na}^{\circ}$ by collecting the large pieces and adding them to hexane. These pieces may have their surface cleaned as described below. The recycled $\mathrm{Na}^{\circ}$ is only employed for THF drying.

\section{Pre-drying of THF}

$\mathrm{NaOH}$ or $\mathrm{KOH}$ (30-35 g; powder, preferentially) was added to a 1L-round bottom flask containing about $900 \mathrm{~mL}$ of THF (CAUTION: THF containing high concentration of peroxides may detonate in presence of such bases). ${ }^{8}$ This mixture in a closed flask was allowed to stand for $48 \mathrm{~h}$. Then, the solvent was distilled slowly under dry $\mathrm{Ar}$ to another round bottom flask. Care was taken to leave about 35 $\mathrm{mL}$ of THF in the flask containing the residue.

\section{Drying of THF}

Thin pieces $(\sim 15 \times 15 \times 2 \mathrm{~mm})^{11}$ of $\mathrm{Na}^{\circ}(\sim 7.0 \mathrm{~g})$, with clean surface, were added to THF $(800-850 \mathrm{~mL})$ in a $1 \mathrm{~L}$-round bottom flask, containing a magnetic bar. The flask was fastened (rubber septum), connected to a system for pressure release (e.g., a balloon or oil bubble) and the mixture was stirred. The metal surface was cleaned periodically by gently pressing metal pieces down with a thick glass stick (CAUTION: The flask bottom should be checked for the presence of cracks before and after this). Stirring continued for 12-24 h. Apparently, evolution of $\mathrm{H}_{2}$ from metal surface had stopped then. If the amount of remaining $\mathrm{Na}^{\circ}$ is considered not sufficient, additional pieces should be added. $\mathrm{Na}^{\circ}$ surface is cleaned once more. Benzophenone ( $1.5 \mathrm{~g}$ ) was added and the flask was flushed with Ar twice, preferentially by a fast flow of gas into the open flask, which was swiftly closed. Then, stirring was promoted. Usually, the color change occurs immediately. In case color change is not observed, additional indicator should be added. Finally, the THF flask was assembled into the still apparatus, which was thoroughly flushed with $\mathrm{Ar}$ (See above). The THF mixture is brought to reflux by means of a heating mantle for the solvent to distill. In order to keep the solvent dry (blue color), one should stir it for 15-20 min every day, after the system has been purged again with Ar. Thorough flushing with Ar is necessary every time the system is charged with either $\mathrm{Na}^{\circ}$ or indicator and before stirring the THF-Na ${ }^{\circ}$ mixture.

\section{SUPPLEMENTARY MATERIAL.}

Available free of charge at http://quimicanova.sbq.org.br as pdf file.

\section{ACKNOWLEDGEMENT}

CNPq and FAPERJ for funding, CAPES for fellowships.

\section{REFERENCES}

1. Schwartz, A. M.; Chem. Eng. News 1978, 24, 88 .

2. http://www.mallbaker.com/techlib/documents/americas/8882.html, accessed March 2009

3. Pangborn, A. B.; Giardello, M. A.; Grubbs, R. H.; Rosen, R. K.; Timmers, F. J.; Organometallics 1996, 15, 1518.

4. A simpler, more affordable modification of the column system (ref. 3) has been proposed. Nevertheless, it has been reported not to work well with THF and other ethereal solvents either: Alaimo, P. J.; Peters, P.W.; Arnold, J.; Bergman, R. G.; J. Chem. Ed. 2001, 78, 64.

5. http://www2.fpm.wisc.edu/chemsafety/research_laboratory_pollution_pr.htm, accessed March 2009.

6. Yarnell, A.; Chem Eng. News 2002, 80, 43.

7. Cournoyer, M. E.; Dare, J. H.; Chem. Health Safety 2003, 15.

8. Coetzee, J. F.; Yang, T. -H.; Pure Appl. Chem. 1985, 57, 633; Org. Synth. Coll. Vol. V, 1973, 976 (General recommendation).

9. Kornblum, N.; Delamare, H.; J. Am. Chem. Soc. 1951, 73, 880.

10. Mete, E.; Altundas, R.; Seçen, H.; Balci, M.; Turk. J. Chem. 2003, 27, 145 , and ref. cited therein.

11. $\mathrm{Na}^{\circ}$ wires may be a convenient alternative. Cleaning of the metal surface during the process still apply, tough. 


\section{AN EXPEDITIOUS AND CONSISTENT PROCEDURE FOR TETRAHYDROFURAN (THF) DRYING AND DEOXYGENATION BY THE STILL APPARATUS}

Alessandro B. C. Simas*, Vera L. P. Pereira, Cleber B. Barreto Jr., Daniel L. de Sales e Leandro L. de Carvalho

Núcleo de Pesquisas de Produtos Naturais, Centro de Ciências da Saúde, bl. H, Universidade Federal do Rio de Janeiro, Ilha do Fundão, 21941-902 Rio de Janeiro - RJ, Brasil
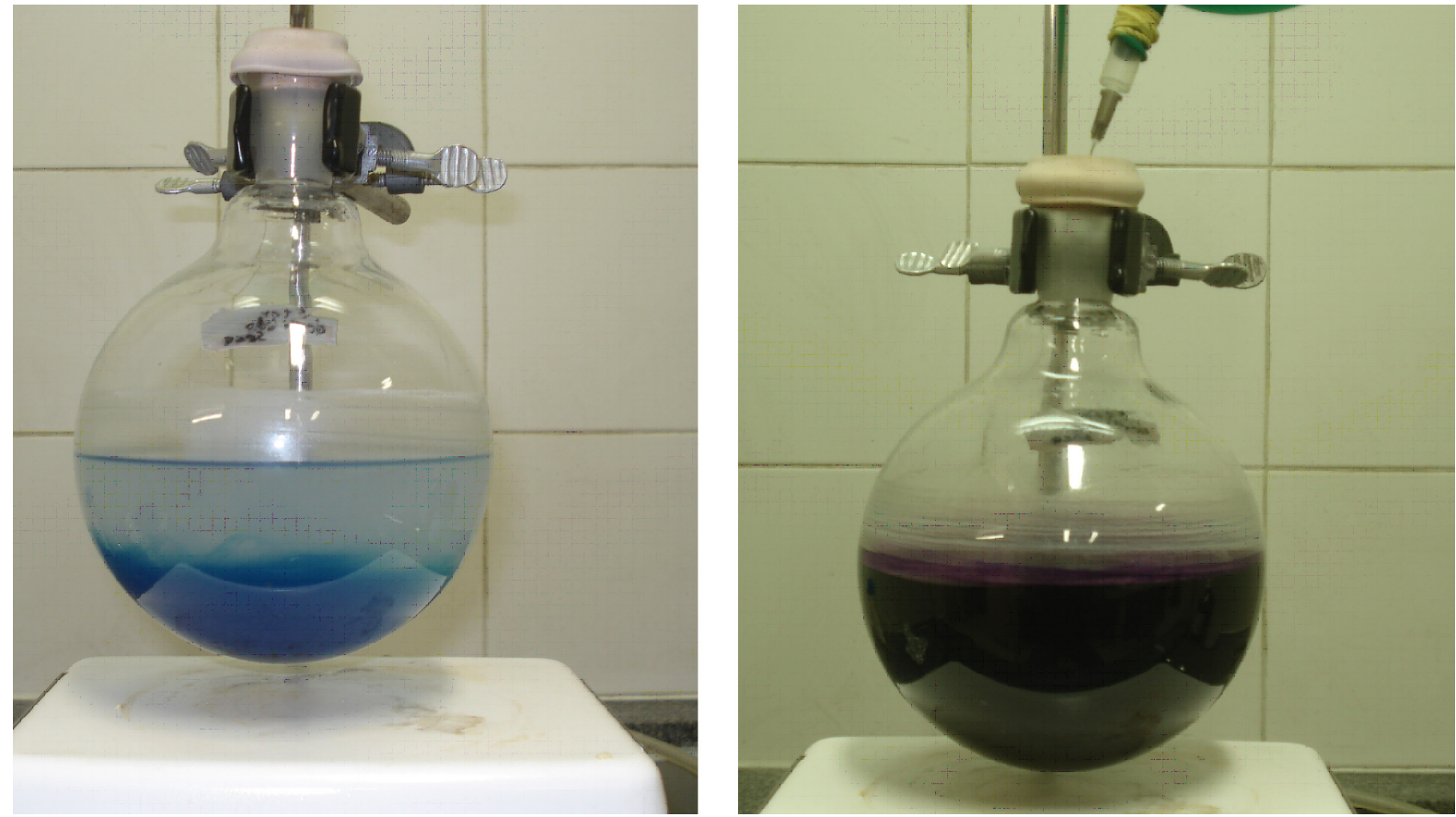

Figure 1S. The benzophenone ketyl-Na indicator in action: Color change in THF as soon as stirring begins (on the left) and THF after continued stirring under Ar (on the right). 